\title{
Kerr and free carrier ultrafast all-optical switching of GaAs/AIAs nanostructures near the three photon edge of GaAs
}

\author{
Alex Hartsuiker, ${ }^{1, a)}$ Philip J. Harding, ${ }^{1}$ Yoanna-Reine Nowicki-Bringuier, ${ }^{2}$ \\ Jean-Michel Gérard, ${ }^{2}$ and Willem L. Vos ${ }^{1,3}$ \\ ${ }^{1}$ Center for Nanophotonics, FOM Institute for Atomic and Molecular Physics (AMOLF), Kruislaan 407, \\ 1098 SJ Amsterdam, The Netherlands \\ ${ }^{2}$ CEA-CNRS Group, Nanophysique et Semiconducteurs, CEA/INAC/SP2M, 17 avenue des Martyrs, \\ 38054 Grenoble Cedex, France \\ ${ }^{3}$ Complex Photonic Systems (COPS), MESA + Institute for Nanotechnology, University of Twente, \\ P.O. Box 217, 7500 AE Enschede, The Netherlands
}

(Received 19 June 2008; accepted 26 August 2008; published online 21 October 2008)

We performed nondegenerate pump-probe experiments on a GaAs/AlAs photonic cavity structure. We switched the photonic properties using the optical Kerr effect and free carriers excited by three photon absorption. The structure was probed at $1150-1640 \mathrm{~nm}$, in the telecom spectral range below the stop gap. In the measurements we observe surprisingly large nondegenerate electronic Kerr coefficients over a broad wavelength range. We also extracted the three photon absorption coefficient for GaAs at three wavelengths in the near infrared. We conclude that the electronic Kerr effect is so large that the resonance of a moderate $Q(Q>1000)$ photonic cavity could be successfully switched instantaneous. (C) 2008 American Institute of Physics.

[DOI: $10.1063 / 1.3000098]$

\section{INTRODUCTION}

Exciting prospects arise when photonic structures are switched on ultrafast time scales. For example, switching would allow the capture or release of photons from photonic band gap cavities, ${ }^{1}$ which is relevant to solid-state slow-light schemes. ${ }^{2}$ Switching the directional properties of photonic crystals also leads to fast changes in the reflectivity, where interesting changes have been reported for Bragg stacks, two-dimensional photonic crystals, ${ }^{5-7}$ first-order stop bands of three-dimensional (3D) opaline crystals, ${ }^{8,9}$ and 3D photonic bandgap crystals. ${ }^{10,11}$ Ultrafast control of the propagation of light is essential to applications in active photonic integrated circuits. ${ }^{12}$

Different mechanisms are possible for switching photonic structures. The switching of photonic structures with free carriers ${ }^{5,6,13,14}$ and a phase transition of $\mathrm{VO}_{2}$ (Ref. 15) have been reported recently. The disadvantage of a phase transition is that the material changes from a transparent material to a metal, which absorbs light. On the other hand free carrier excitation is ultrafast, but the recombination of the carriers limits the switch-off time to a few picoseconds time scale.

With instantaneous switching it would be possible to ultimately control both the on- and off-times of the switching event. This is possible with the electronic Kerr effect, where the on- and off-times are determined by the pulse duration, which is in the order of femtoseconds rather than picoseconds as with free carrier switching. This extreme of fast switching could be used for capturing and releasing photons on demand, for example, in a vertical-cavity surface emitting laser. $^{16}$

\footnotetext{
${ }^{a)}$ Electronic mail: hartsuiker@amolf.nl.
}

In order to switch a cavity, the refractive index change induced with the electronic Kerr effect should be large enough. To shift the cavity resonance by one linewidth the refractive index change should be equal to $\left(\Delta n^{\prime} / n^{\prime}\right)$ $=(1 / Q) .{ }^{10}$ Thus for an experiment with a cavity having a quality factor $Q$ of 1000 , this means a refractive index change of $0.1 \%$. It is widely assumed (see Ref. 1) that the magnitude of the refractive index change by the Kerr effect is much smaller than the change due to a free carrier switch. Therefore most switching schemes use free carriers and are therefore limited to picosecond time scales.

A problem which occurs at coincidence of pump and probe pulse is nondegenerate two photon absorption. ${ }^{13}$ Here, a probe photon is absorbed together with a pump photon when the summed energy of the pump and probe exceed the bandgap energy. The change in imaginary part of the refractive index can be large compared to the change in the real part, giving rise to absorptive changes in the optical properties.

In this work we demonstrate a method to decrease nondegenerate two photon absorption at pump and probe coincidence. Because of this, we observe that the electrical Kerr effect is surprisingly large and competitive with free carrier effects. We conclude that a cavity resonance with a moderate $Q$ of 1000 or higher could be successfully switched instantaneously. We derive the Kerr coefficient and the three photon absorption coefficient over a broad wavelength range.

\section{EXPERIMENTAL}

\section{A. Sample}

Our structure consists of a GaAs $\lambda$ thick layer $(277 \mathrm{~nm}$ thick) sandwiched between two Bragg stacks consisting of 12 and 16 pairs of $\lambda / 4$ thick layers of nominally pure GaAs 
or AlAs. The same structure was studied in Ref. 13. The sample is grown with molecular beam epitaxy at $550{ }^{\circ} \mathrm{C}$ to optimize the optical quality. ${ }^{17}$ For experiments outside the present scope the sample was doped with $10^{10} \mathrm{~cm}^{-2} \mathrm{InGaAs} /$ GaAs quantum dots, which hardly influence our experiment. $^{18}$

\section{B. Optical measurements}

Our setup consists of two independently tunable optical parametric amplifiers (OPAs) (Topas) that are the sources of the pump and probe beams. The setup has been described in Ref. 19, therefore we present only a brief outline here. The OPAs have pulse durations $\tau_{P}=140 \pm 10$ fs. The pump beam has a much larger Gaussian focus of $113 \mu \mathrm{m}$ full width at half maximum than the probe beam $(28 \mu \mathrm{m})$, ensuring that only the central flat part of the pump focus is probed. A versatile measurement scheme was developed to subtract the pump background from the probe signal, and to compensate for possible pulse-to-pulse variations in the output of our laser. ${ }^{19}$ Separately, continuous-wave (cw) reflectivity was measured with a broadband white light setup with a resolution of $\sim 0.2 \mathrm{~nm}^{20}$

In this work we varied the pump wavelength from 2000 to $2400 \mathrm{~nm}$ in steps of $200 \mathrm{~nm}$. The probe wavelength was varied independently of the pump wavelength between 1150 and $1650 \mathrm{~nm}$, with steps of $5 \mathrm{~nm}$. Measured data were corrected for dispersion in the system.

\section{RESULTS}

\section{A. Linear reflectivity}

Figure 1(c) shows the measured linear reflectivity spectrum of our sample and a transfer matrix (TM) calculation. ${ }^{21}$ The TM calculation including the dispersion of GaAs (Ref. 22) and AlAs (Ref. 23) reproduces the experimental resonance, stop band, and Fabry-Pérot fringes. The only free parameters in the model were the thicknesses of the GaAs $(d \mathrm{GaAs}=69.2 \mathrm{~nm})$ and AlAs $(d \mathrm{AlAs}=81.0 \mathrm{~nm})$, which agree to the measured values. The reflectivity spectrum shows the stop band with cavity resonance and Fabry-Pérot fringes. The spectrum can be divided into two spectral regions, namely, "absorptive" (right, densely hatched) and "dispersive" (left, hatched), referring to the expected behavior if pump and probe coincide.

The origin of the absorption is explained in Figs. 1(a) and 1(b), which show energy schematics at coincidence of pump and probe. In the densely hatched spectral region, the pump and probe photon will be absorbed since the sum of the photon energies is higher than the bandgap of GaAs. In the sparsely hatched (dispersive) spectral region however, the sum of pump and probe photon energies is below the bandgap of GaAs. Therefore absorption is low in this region and behavior at coincidence is therefore mainly dispersive. Only the real part of the refractive index $n^{\prime}$ changes. The regions are separated at a probe wavelength which corresponds to $1340 \mathrm{~nm}$ at a pump wavelength of $2400 \mathrm{~nm}$.

The origin of the dispersion is the electronic Kerr effect. There will be a change in the real part of the refractive index $\left(n^{\prime}\right)$ due to the pump field. The edge of the hatched regions

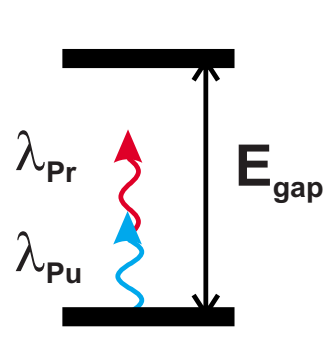

(a)

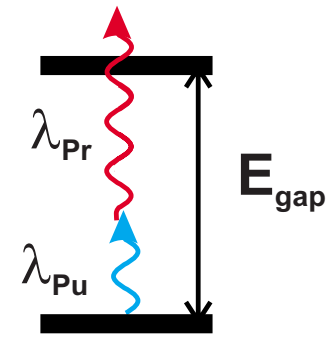

(b)

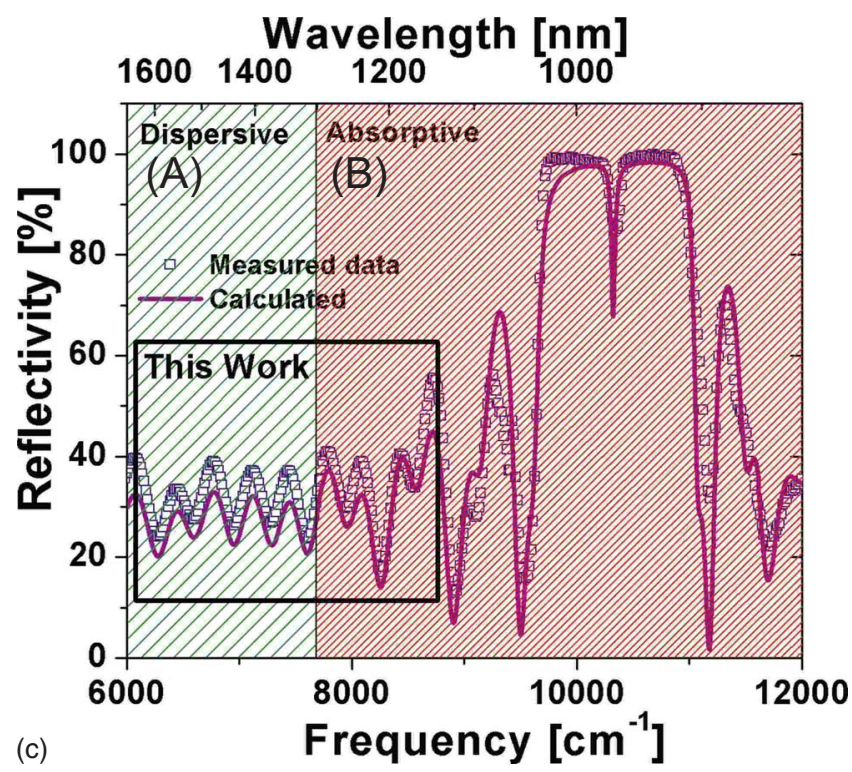

FIG. 1. (Color online) [(a) and (b)] Energy schematics of GaAs. $E_{\text {gap }}$ is the electronic bandgap of GaAs, $\lambda_{P r}$ is the probe wavelength and $\lambda_{P u}$ is the pump wavelength. (a) In the dispersive spectral region the summed energy of a pump and a probe photon is smaller than the bandgap, while (b) the summed energy is larger in the absorptive spectral region. The edge between diagrams (a) and (b) is at probe wavelengths of 1510 and 1340 at pump wavelengths of 2000 and $2400 \mathrm{~nm}$, respectively. We present measurements in the spectral region indicated with a square, to obtain a change in the real part of the refractive index. (c) Linear reflectivity spectrum and TM calculation of the GaAs/AlAs structure. The trough at $980 \mathrm{~nm}$ is due to the cavity resonance of the lambda thick GaAs layer. The hatched regions are based on a pump wavelength of $2400 \mathrm{~nm}$. The slight difference in amplitude of the measured and calculated reflectivity on the red side of the stop band is caused by a small error in the normalization measurement.

in Fig. 1(c) will shift to the red at a pump wavelength of $2000 \mathrm{~nm}$, indicating that we expect absorption in a large part of the spectrum. The behavior at coincidence is therefore mainly absorptive, meaning a change in the imaginary part of the refractive index $\left(n^{\prime \prime}\right)$.

We focus in this work on the spectral region indicated by the black rectangle. In this region we expect to observe effects of the optical Kerr effect but only little absorption at $2400 \mathrm{~nm}$ pump wavelength, while we expect mainly absorption at $2000 \mathrm{~nm}$ pump wavelength.

\section{B. Ultrafast switched reflectivity}

The two plots in Fig. 2 show the differential reflectivity measured at $2000 \mathrm{~nm}, I_{\text {pump }}=90 \mathrm{GW} / \mathrm{cm}^{2}$ (a) and $2400 \mathrm{~nm}$, $I_{\text {pump }}=95 \mathrm{GW} / \mathrm{cm}^{2}$ (c) pump wavelength as a function of probe wavelength and delay. Cross sections of Figs. 2(a) and 2(c) are given in Figs. 2(b) and 2(d), respectively. 

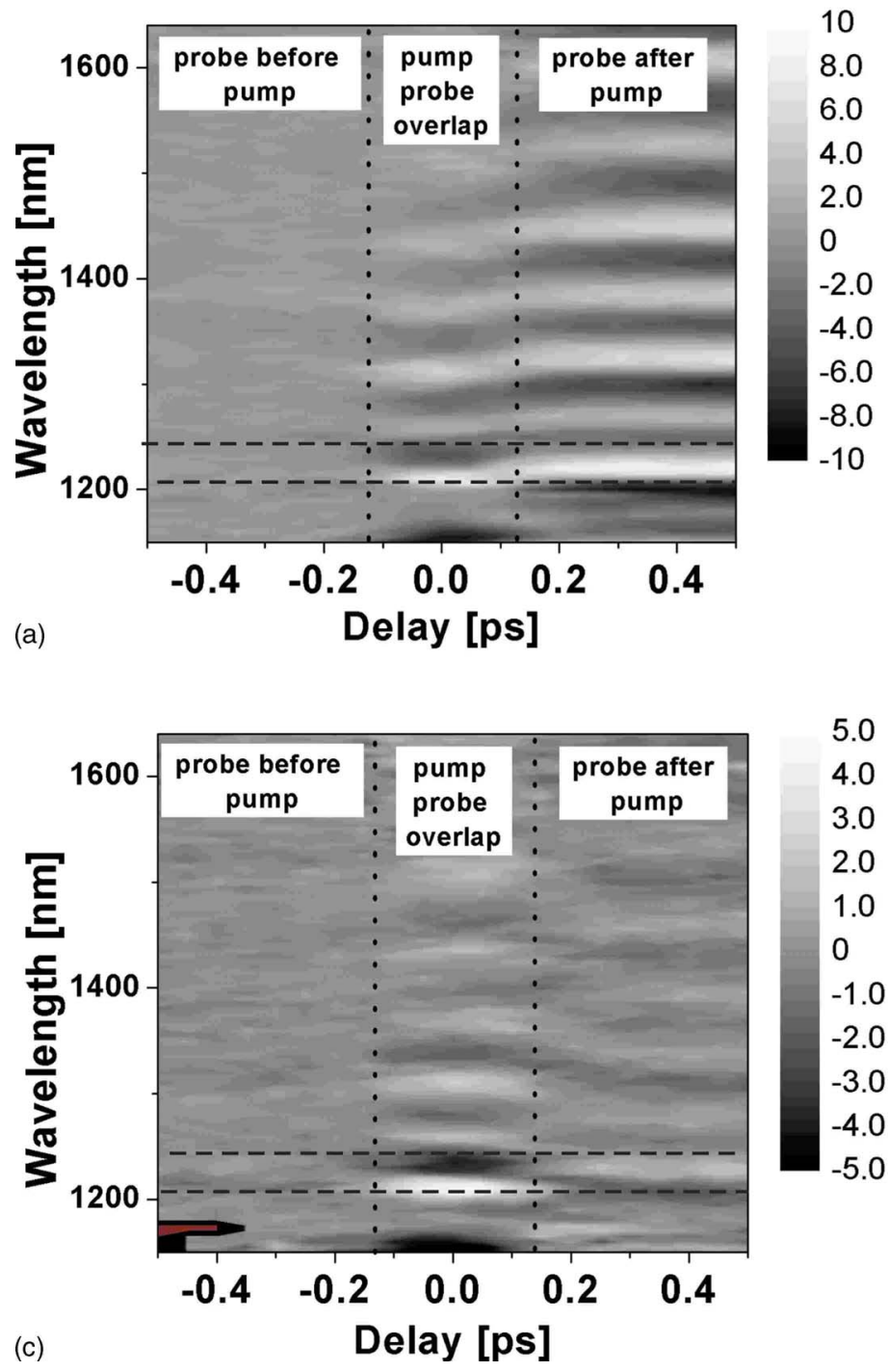
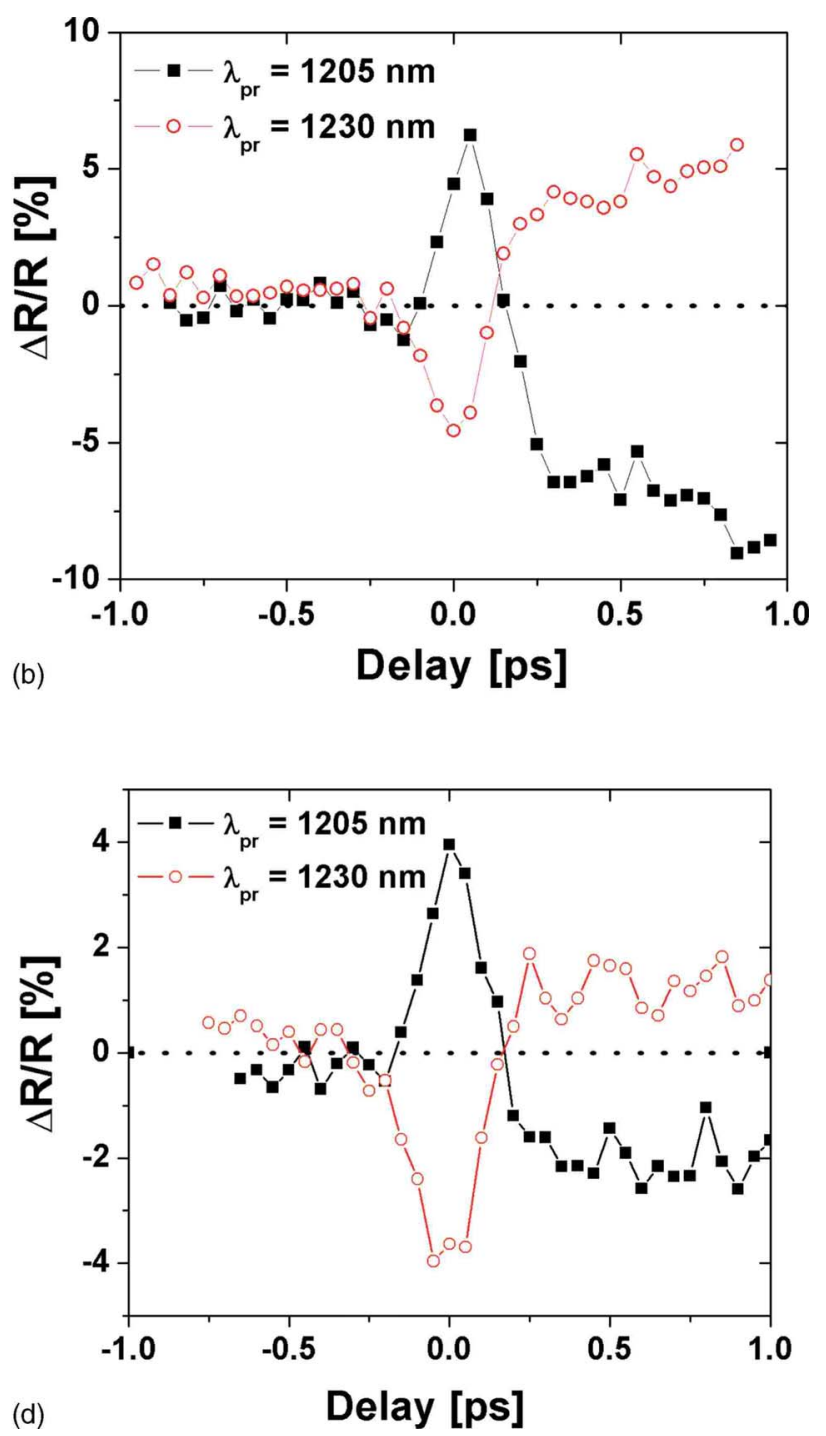

FIG. 2. (Color online) Differential reflectivity per wavelength as a function of delay between pump and probe pulse. At negative delays the pump hits the sample before the probe. The scans were measured at different pump wavelengths. (a) $\lambda_{\text {pump }}=2000 \mathrm{~nm}, I_{\text {pump }}=90 \mathrm{GW} / \mathrm{cm}^{2},(\mathrm{c}) \lambda_{\text {pump }}=2400 \mathrm{~nm}, I_{\text {pump }}$ $=95 \mathrm{GW} / \mathrm{cm}^{2}$. Cross cuts indicated by dashed lines in (a) and (c) are shown in (b) and (d), respectively. (a) and (c) show a fringe pattern indicating a shift of the Fabry-Pérot fringes. Cross sections (b) and (d) show that the sign of the differential reflectivity at coincidence is different from the sign at positive delay.

A fringe pattern is visible in Figs. 2(a) and 2(c) for coincidence of pump and probe and for positive delay. Figures 2(b) and 2(d) show cross sections of Figs. 2(a) and 2(c), respectively. The sign difference in differential reflectivity between pump and probe overlap and positive delay is apparent and is a result from the fringe patterns at coincidence and positive delay, which are spectrally shifted with respect to each other. This indicates that the switch mechanism at coincidence is different from the free carrier mechanism at positive delay. Figure 2(d) shows that there is a competition between the instantaneous effect and the free carrier effect.

At a pump wavelength of $2000 \mathrm{~nm}$ we expect a change in $n^{\prime \prime}$ since we are in the absorptive regime. At $2400 \mathrm{~nm}$ pump wavelength we expect a change in $n^{\prime}$ and a negligible change in $n^{\prime \prime}$ since we are in the dispersive region (see Fig. 1). The differential reflectivity at positive delay is caused by excited free carriers. Figure 3 shows the power dependence of differential reflectivity $(\Delta R / R)$ at positive delay.
There is a linear relation between the differential reflectivity and the power cubed, as shown in Fig. 3. We therefore conclude that the differential reflectivity caused by free carriers is generated by a three photon absorption process.

\section{Interpretation of time-resolved reflectivity}

The fringe pattern in Figs. 2(a) and 2(c) results from Fabry-Pérot interferences within the stack forming the optical microcavity. A change in $n^{\prime}$ modifies the optical thickness of the GaAs layers; it induces a spectral shift of the Fabry-Pérot fringes, which results in a periodic differential reflectivity. A change in $n^{\prime \prime}$ decreases the modulation depth of the Fabry-Pérot fringes.

The differential reflectivity due to a change in the real part of the refractive index depends on measured intensities as follows: 


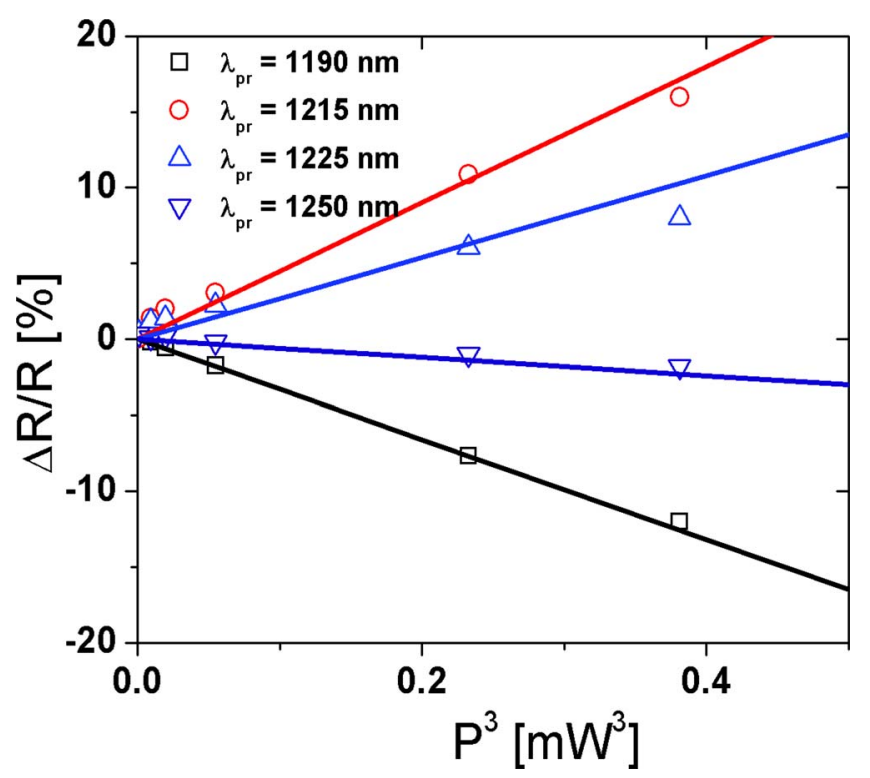

FIG. 3. (Color online) Differential reflectivity at positive delay measured at different probe wavelengths at a pump wavelength of $2000 \mathrm{~nm}$. The differential reflectivity is plotted as a function of pump power cubed. The relation between the differential reflectivity at positive delay and the power cubed is linear. We conclude that the carriers are solely generated through a three photon process.

$$
\frac{\Delta R}{R}\left(\tau, \omega_{\mathrm{pr}}, \omega_{\mathrm{pu}}\right)=\frac{I_{\mathrm{pu}}\left(\tau, \omega_{\mathrm{pr}}, \omega_{\mathrm{pu}}\right)-I_{\mathrm{unpu}}\left(\omega_{\mathrm{pr}}\right)}{I_{\mathrm{unpu}}\left(\omega_{\mathrm{pr}}\right)},
$$

where $\Delta R / R$ is the differential reflectivity, $\tau$ is the delay between pump and probe, $\omega_{\mathrm{pr}}$ is the probe frequency, $\omega_{\mathrm{pu}}$ is the pump frequency, $I_{\text {unpu }}$ is the reflectance measured if the structure is unpumped, and $I_{\mathrm{pu}}$ is the reflectance measured if the structure is pumped.

In case of a change in the real part of the refractive index, the fringe pattern shifts spectrally $\Delta \omega_{\mathrm{pr}}=\alpha n^{\prime}$, with $\alpha$ a proportionality constant that depends on the exact structure of the fringe pattern. $I_{\mathrm{pu}}$ is then given by

$$
I_{\mathrm{pu}}\left(\tau, \omega_{\mathrm{pr}}, \omega_{\mathrm{pu}}\right)=I_{\mathrm{unpu}}\left[\omega_{\mathrm{pr}}+\Delta \omega_{\mathrm{pr}}\left(\tau, \omega_{\mathrm{pu}}\right)\right],
$$

which can be written as

$$
I_{\mathrm{pu}}\left(\tau, \omega_{\mathrm{pr}}, \omega_{\mathrm{pu}}\right)=I_{\mathrm{unpu}}\left(\omega_{\mathrm{pr}}\right)+\frac{\partial I_{\mathrm{unpu}}\left(\omega_{\mathrm{pr}}\right)}{\partial \omega_{\mathrm{pr}}} \Delta \omega_{\mathrm{pr}}\left(\tau, \omega_{\mathrm{pu}}\right),
$$

which yields a differential reflectivity,

$$
\frac{\Delta R}{R}\left(\tau, \omega_{\mathrm{pr}}, \omega_{\mathrm{pu}}\right)=\frac{1}{I_{\mathrm{unpu}}\left(\omega_{\mathrm{pr}}\right)} \frac{\partial I_{\mathrm{unpu}}\left(\omega_{\mathrm{pr}}\right)}{\partial \omega_{\mathrm{pr}}} \Delta \omega_{E}\left(\tau, \omega_{\mathrm{pu}}\right) .
$$

Equation (4) shows that in the case of a change in the real part of the refractive index a large differential reflectivity will be measured where the unpumped reflectivity has a large derivative, which is spectrally between successive maxima and minima of the Fabry-Pérot fringes. This is illustrated in Fig. 4. Figure 4 shows a TM calculation using parameters relevant to our structure. In the case of a change in $n^{\prime \prime}$ the modulation depth of the fringe pattern is affected, while in the case of a change in $n^{\prime}$ the position of the fringe pattern is shifted. For this reason the differential reflectivity has maxima at different positions making it possible to distin-
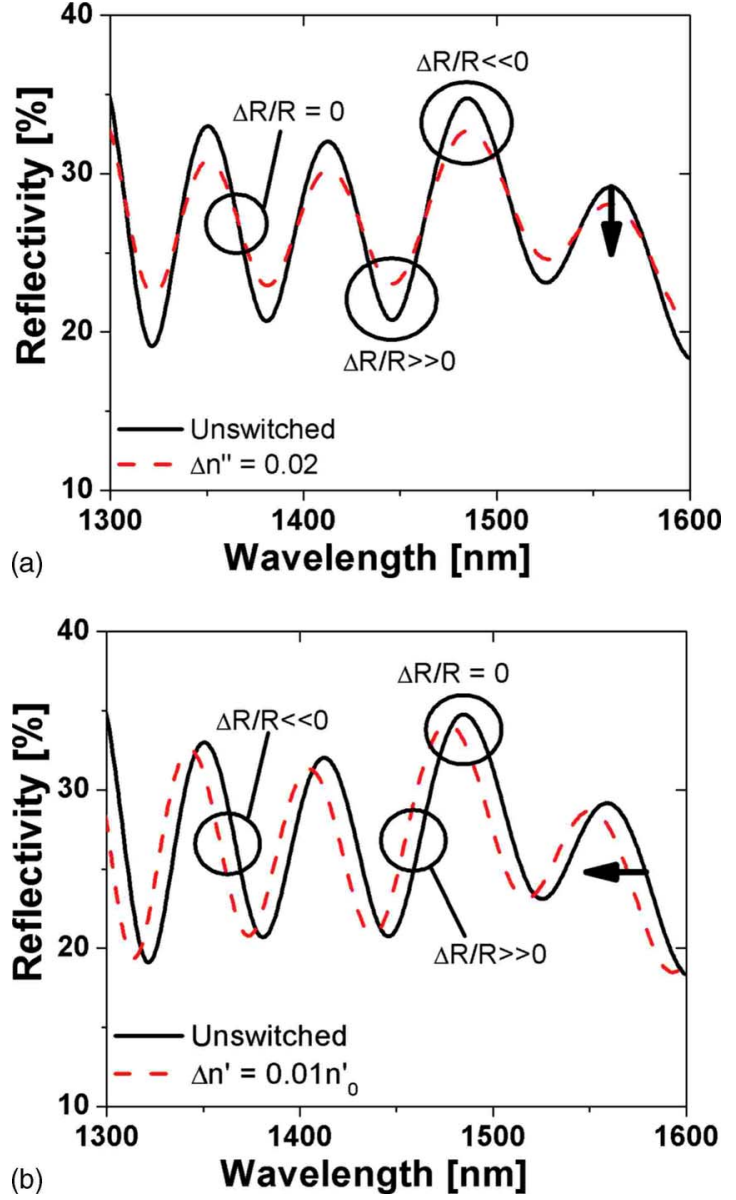

FIG. 4. (Color online) Calculated switched and unswitched reflectivity for a change in the $n^{\prime \prime}$ (a) and a change in $n^{\prime}$ (b). The calculation was done with a TM model using parameters relevant to our structure. (a) shows that the introduction of absorption mainly affects the modulation depth of the fringes. A change in $n^{\prime}$ causes a shift of the fringe pattern (b). The differential reflectivity has maxima at different spectral positions, which makes it possible to distinguish between a purely dispersive and a purely absorptive regime.

guish between a purely dispersive and a purely absorptive regime.

Figure 5 shows a TM calculation of the differential reflectivity spectrum of the planar microcavity. The intensity profile in the structure is homogeneous, leading to a homogeneous refractive index change. The intensity profile is homogenous since we pump at a long wavelength in the three photon absorption regime. The homogeneity length ${ }^{24}$ is longer than our sample.

Figure 5 shows a comparison between the measured and calculated differential reflectivity pattern. Comparing the measured and calculated patterns shows that the positions of the peaks and troughs in the differential reflectivity pattern near zero delay are caused by a change in the imaginary part of the refractive index $\left(n^{\prime \prime}\right)$ in the case of $2000 \mathrm{~nm}$ pump and a change in the real part of the refractive index $\left(n^{\prime}\right)$ in the case of $2400 \mathrm{~nm}$ pump. We conclude that in the case of 2400 $\mathrm{nm}$ pump wavelength the fringe pattern near zero delay in the differential reflectivity originates from an electronic Kerr switch since the process is ultrafast, instantaneous with the laser pulse and is dispersive. Interestingly there is a competition between the Kerr and free carrier effects, with the Kerr 

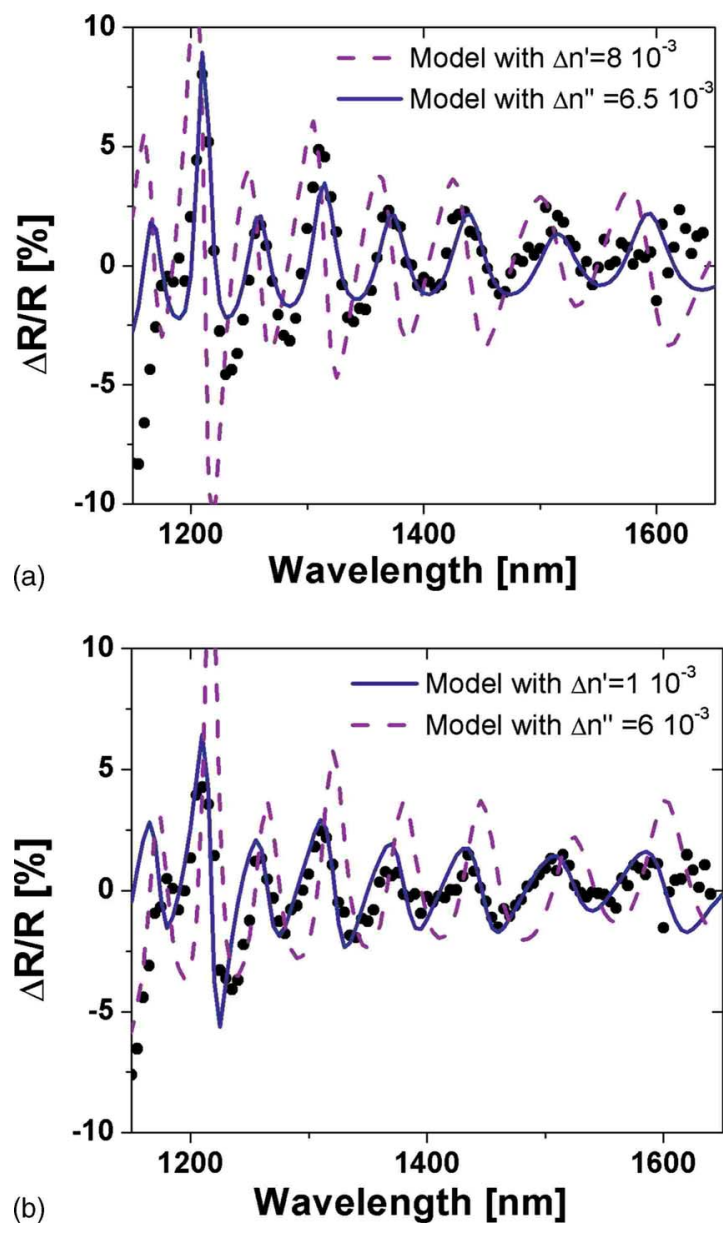

FIG. 5. (Color online) Cross section of Fig. 2 (black solid circles) showing the differential reflectivity $\Delta R / R$ as a function of probe wavelength at pump-probe coincidence $(\Delta t=0 \mathrm{ps})$. The structure was pumped at $2000 \mathrm{~nm}$ (a) and $2400 \mathrm{~nm}$ (b). The solid and dashed lines are results from TM calculations. In (a) the dashed line represents a change in the real part of the refractive index while the solid line represents a change in the imaginary part of the refractive index. This is the other way around in (b): dashed represents a change in imaginary part, while solid represents a change in the real part of refractive index. As expected we see mainly a change in the imaginary part of the refractive index at $2000 \mathrm{~nm}$ pump and a change in $n^{\prime}$ at $2400 \mathrm{~nm}$. Furthermore our model slightly deviates near the blue side of the spectrum.

effect responsible for a $\Delta n^{\prime}>0$ and the free carrier effect for a $\Delta n^{\prime}<0$. Surprisingly the electronic Kerr effect is larger than the free carrier effect in this wavelength range. A comparable result to the present GaAs results has recently been obtained for Si photonic crystals. ${ }^{25}$

In case of $2000 \mathrm{~nm}$ pump wavelength the fringe pattern originates from nondegenerate two photon absorption. Furthermore we see a slight deviation of our model near the blue side of the spectrum, indicating some dispersion in the nonlinear effect. We further conclude from Fig. 5 that absorption can be neglected at $2400 \mathrm{~nm}$ pump wavelength, while dispersion can be neglected at $2000 \mathrm{~nm}$ pump wavelength.

By varying the size of the change in $n^{\prime}$ we extracted the change in refractive index at each wavelength. The results are plotted together with dispersion curves in Fig. 6. Figure 6 shows no change in refractive index for a negative delay (a), a positive change for zero delay (b), and a negative change for positive delay (c). Furthermore there is a good agreement
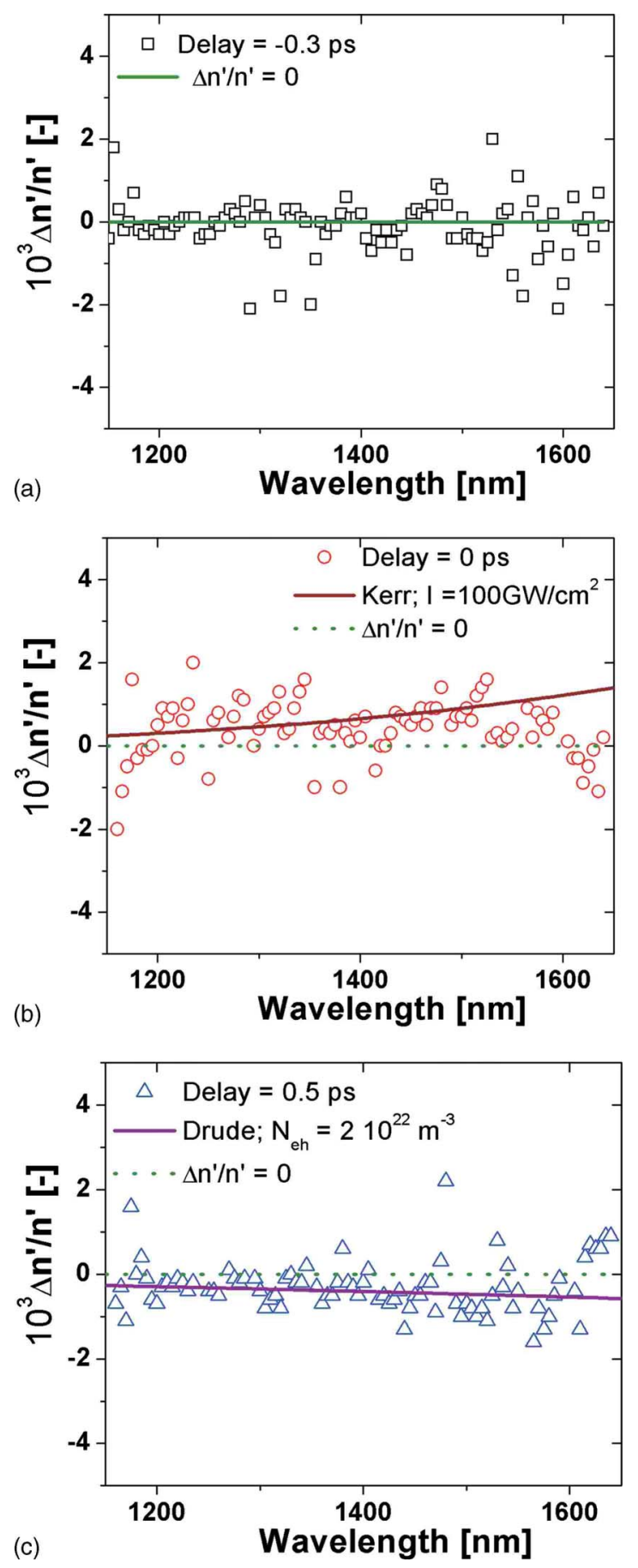

FIG. 6. (Color online) Relative change of refractive index due to a $2400 \mathrm{~nm}$ pump as a function of probe wavelength at a delay of (a) $-0.3 \mathrm{ps}$, (b) $0 \mathrm{ps,}$ and (c) 0.5 ps. The dashed line in all three cases represent no change in refractive index. The solid line in (b) represents the dispersion of the change in refractive index from Ref. 26. The solid line in (c) is calculated with the Drude model for free carriers (Ref. 19). Points obtained from spectral regions close to extreme of the fringes were removed because of their poor precision.

between the data and the dispersion of the electronic Kerr effect $^{26}$ and the dispersion of the free carrier excitation. ${ }^{19}$

The scattered symbols deviating strongly from the theoretical model can be attributed to small differences in shape of the measured and calculated reflectivity spectrum. A slight deviation is amplified by the fitting procedure since we extract the refractive index change by fitting the differential reflectivity at one wavelength. 


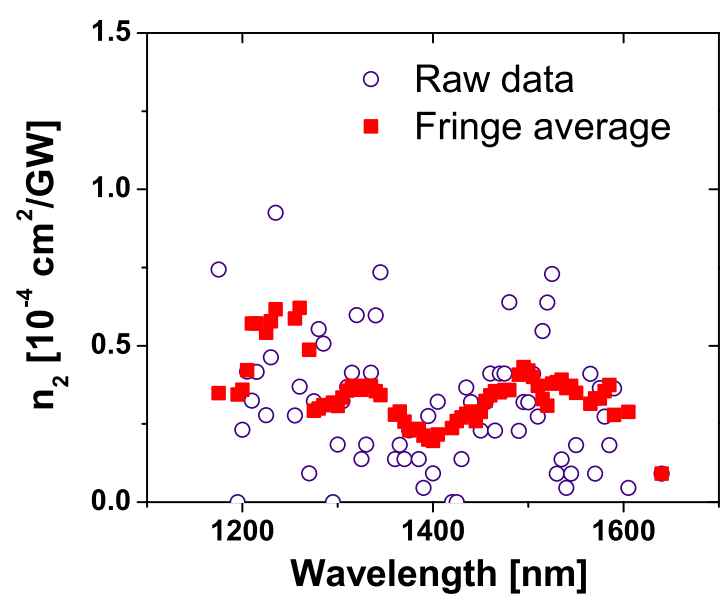

FIG. 7. (Color online) Measured nondegenerate Kerr coefficient $n_{2}$ as a function of probe wavelength (open circles). We averaged the data over the period of one fringe since the coefficients are correlated within this fringe period (solid squares). We observe dispersion in $n_{2}$ toward the blue side of the spectrum as expected from Fig. 5.

Figure 6(b) shows that the change in refractive index induced by the optical Kerr effect is in the order of $0.1 \%$. This is large enough to switch a cavity with a moderate $Q$ of 1000. From our experiments two consequences follow for the design of a GaAs cavity that can be Kerr switched. First the cavity should be pumped at $2400 \mathrm{~nm}$ of higher. Furthermore the resonance of the cavity should be in the spectral region of $1300 \mathrm{~nm}$ or higher [see Fig. 1(c)].

\section{Nonlinear coefficients GaAs}

\section{Kerr coefficient $n_{2}$ for GaAs}

The nondegenerate Kerr coefficient $n_{2}$ can directly be extracted from the data $\Delta n^{\prime}$ in Fig. 6 using the relation, ${ }^{26,27}$

$$
n_{2}=\frac{\Delta n^{\prime}}{2 I_{0}}
$$

where $I_{0}$ is the pump intensity. Since $n_{2}$ scales with $E_{\text {gap }}^{-4}{ }^{26}$ the contribution of AlAs is only $20 \%$ of the total $n_{2}$. For simplicity we therefore assume that mainly the GaAs is switched.

The resulting Kerr coefficients are plotted in Fig. 7. Figure 7 shows the raw data extracted from Fig. 6 (open circles) and the data averaged over the width of a fringe (solid squares). The order of magnitude of $n_{2}$ is $10^{-4} \mathrm{GW} \mathrm{cm}^{-2}$. The nondegenerate Kerr coefficient decreases with increasing wavelength. This dispersive behavior was already observed in Fig. 5.

The values of $n_{2}$ measured in our nondegenerate pumpprobe experiment are similar to the ones reported in literature for degenerate pump-probe experiments. ${ }^{28-30} \mathrm{We}$ find this surprising since we pump at a wavelength twice as long as in the degenerate case. This result enables us to modify efficiently the refractive index with a far detuned pump wavelength.

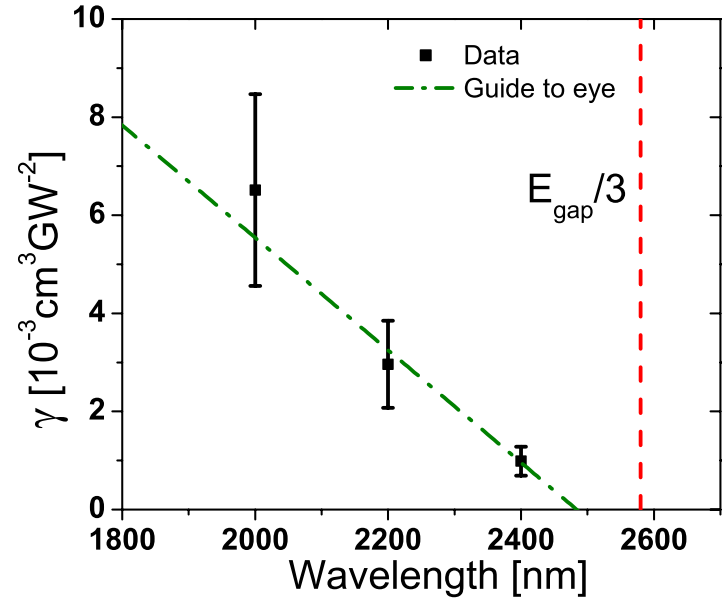

FIG. 8. (Color online) Three photon absorption coefficient as a function of wavelength extracted from the differential reflectivity data. The relative error of $30 \%$ is indicated.

\section{Three photon absorption coefficient $\gamma$ for GaAs}

Figure 6 shows that we are able to derive a carrier density from the measured change in refractive index with the use of the Drude model. We will discuss in this section the three photon absorption coefficient $\gamma$.

Figure 8 shows the three photon absorption coefficient $\gamma$ as a function of pump wavelength. The order of magnitude of $\gamma$ is $10^{-3} \mathrm{~cm}^{3} \mathrm{GW}^{-2}$. Our data are two orders of magnitude smaller than the values reported in literature, ${ }^{31}$ measured with the $z$-scan method. We attribute this difference to the fact that our data have been obtained on an epitaxially grown GaAs/AlAs heterostructure instead of a GaAs wafer obtained with a different technique.

The three photon absorption coefficient decreases as a function of wavelength since the summed energy of a pump and probe photon, and therefore the probability of generating an electron hole pair, decreases with increasing wavelength. The three photon absorption edge is $2580 \mathrm{~nm}$.

\section{CONCLUSION}

We studied ultrafast all-optical switching of the fringes of a GaAs/AlAs planar optical microcavity. We demonstrate conditions where the instantaneous electronic Kerr effect prevails over two photon absorption. Contrary to prior expectations, we find that the electronic Kerr effect is even larger than free carrier effects.

From the switching measurements we extracted the nondegenerate Kerr coefficients over a broad wavelength range. Furthermore we extracted the free carrier three photon absorption coefficients for three different wavelengths in the near infrared.

We conclude from our results that it is possible to switch a GaAs cavity with a $Q$ higher than 1000 , using the electronic Kerr effect. For such an experiment a pump wavelength of $2400 \mathrm{~nm}$ or larger is required and a cavity resonance at a wavelength in the telecom spectral range of 1300 $\mathrm{nm}$ or larger. The refractive index change is in the order of the required $0.1 \%$. 


\section{ACKNOWLEDGMENTS}

We want to thank Allard Mosk and Patrick Johnson for stimulating discussion. This research was supported by NanoNed, a nanotechnology program of the Dutch Ministry of Economic Affairs, and by a VICI fellowship from the "Nederlandse Organisatie voor Wetenschappelijk Onderzoek" (NWO) to WLV. This work is also part of the research program of the "Stichting voor Fundamenteel Onderzoek der Materie" (FOM), which is financially supported by the NWO.

${ }^{1}$ P. M. Johnson, A. F. Koenderink, and W. L. Vos, Phys. Rev. B 66, 081102(R) (2002).

${ }^{2}$ M. F. Yanik and S. Fan, Phys. Rev. Lett. 92, 083901 (2004).

${ }^{3}$ A. Haché and M. Bourgeois, Appl. Phys. Lett. 77, 4089 (2000).

${ }^{4}$ S. R. Hastings, M. J. A. de Dood, H. Kim, W. Marshall, H. S. Eisenberg, and D. Bouwmeester, Appl. Phys. Lett. 86, 031109 (2005).

${ }^{5}$ S. W. Leonard, H. M. van Driel, J. Schilling, R. B. Wehrspohn, Phys. Rev. B 66, 161102(R) (2002).

${ }^{6}$ H. W. Tan, H. M. van Driel, S. L. Schweizer, R. B. Wehrspohn, and U. Gösele, Phys. Rev. B 70, 205110 (2004).

${ }^{7}$ A. D. Bristow, J.-P. R. Wells, W. H. Fan, A. M. Fox, M. S. Skolnick, D. M. Whittaker, A. Tahraoui, T. F. Krauss, and J. S. Roberts, Appl. Phys. Lett. 83, 851 (2003)

${ }^{8}$ D. A. Mazurenko, R. Kerst, J. I. Dijkhuis, A. V. Akimov, V. G. Golubev, D. A. Kurdyukov, A. B. Pevtsov, and A. V. Sel'kin, Phys. Rev. Lett. 91, 213903 (2003)

${ }^{9}$ C. Becker, S. Linden, G. von Freymann, M. Wegener, N. Tétreault, E. Vekris, V. Kitaev, and G. A. Ozin, Appl. Phys. Lett. 87, 091111 (2005).

${ }^{10}$ T. G. Euser, A. J. Molenaar, J. G. Fleming, B. Gralak, A. Polman, and W. L. Vos, Phys. Rev. B 77, 115214 (2008).

${ }^{11}$ T. G. Euser, H. Wei, J. Kalkman, Y. Jun, A. Polman, D. J. Norris, and W. L. Vos, J. Appl. Phys. 102, 053111 (2007).
${ }^{12}$ H. Nakamura, Y. Sugimoto, K. Kanamoto, N. Ikeda, Y. Tanaka, Y. Nakamura, S. Ohkouchi, Y. Watanabe, K. Inoue, H. Ishikawa, and K. Asakawa, Opt. Express 12, 6606 (2004).

${ }^{13}$ P. J. Harding, T. G. Euser, Y.-R. Nowicki-Bringuier, J.-M. Gérard, and W. L. Vos, Appl. Phys. Lett. 91, 111103 (2007).

${ }^{14}$ X. Hu, P. Jiang, C. Ding, H. Yang, and Q. Gong, Nat. Photonics 2, 185 (2008).

${ }^{15}$ D. A. Mazurenko, R. Kerst, J. I. Dijkhuis, A. V. Akimov, V. G. Golubev, A. A. Kaplyanskii, D. A. Kurdyukov, and A. B. Pevtsov, Appl. Phys. Lett. 86, 041114 (2005).

${ }^{16}$ S. G. Hense and M. Wegener, Phys. Rev. B 55, 9255 (1997).

${ }^{17}$ J.-M. Gérard, D. Barrier, J. Y. Marzin, R. Kuszelewicz, L. Manin, V. Costard, V. Thierry-Mieg, and T. Rivera, Appl. Phys. Lett. 69, 449 (1996).

${ }^{18}$ The maximum unbroadened refractive index change of the dots amounts to only $10^{-8}$, while the absorption at resonance is less than $0.02 \mathrm{~cm}^{-1}$.

${ }^{19}$ T. G. Euser, Ph.D. thesis, University of Twente, 2007.

${ }^{20}$ M. S. Thijssen, R. Sprik, J. E. G. J. Wijnhoven, M. Megens, T. Narayanan, A. Lagendijk, and W. L. Vos, Phys. Rev. Lett. 83, 2730 (1999).

${ }^{21}$ M. Born and E. Wolf, Principles of Optics: Electromagnetic Theory of Propagation, Interference and Diffraction of Light (Cambridge University Press, Cambridge, 1999).

${ }^{22}$ J. S. Blakemore, J. Appl. Phys. 53, R123 (1982).

${ }^{23}$ R. E. Fern and A. Onton, J. Appl. Phys. 42, 3499 (1971).

${ }^{24}$ T. G. Euser and W. L. Vos, J. Appl. Phys. 97, 043102 (2005).

${ }^{25}$ P. J. Harding, T. G. Euser, and W. L. Vos, e-print arXiv:0807.4459.

${ }^{26}$ M. Sheik-Bahae, J. Wang, and E. W. van Stryland, IEEE J. Quantum Electron. 30, 249 (1994).

${ }^{27}$ R. W. Boyd, Nonlinear Optics (Academic, New York, 1992), p. 162.

${ }^{28}$ M. Dinu, F. Quochi, and H. Garcia, Appl. Phys. Lett. 82, 2954 (2003).

${ }^{29}$ T. G. Ulmer, R. K. Tan, Z. Zhou, S. E. Ralph, R. P. Kenan, and C. M. Verber, Opt. Lett. 24, 756 (1999).

${ }^{30}$ A. A. Said, M. Sheik-Bahae, D. J. Hagan, T. H. Wei, J. Wang, J. Young, and E. W. Van Stryland, J. Opt. Soc. Am. B 9, 405 (1992).

${ }^{31}$ W. C. Hurlbut, Y.-S. Lee, K. L. Vodopyanov, P. S. Kuo, and M. M. Fejer, Opt. Lett. 32, 668 (2007). 